\title{
AUTOMATIC CONTINUITY OF CERTAIN ISOMORPHISMS BETWEEN REGULAR BANACH FUNCTION ALGEBRAS
}

\author{
by JUAN J. FONT $\dagger$
}

(Received 9 May, 1996)

\begin{abstract}
Let $A$ and $B$ be regular semisimple commutative Banach algebras; that is to say, regular Banach function algebras. A linear map $T$ defined from $A$ into $B$ is said to be separating or disjointmess preserving if $f . g \equiv 0$ implies $T f . T g \equiv 0$, for all $f, g \in A$. In this paper we prove that if $A$ satisfies Ditkin's condition then a separating bijection is automatically continuous and its inverse is separating. If also $B$ satisfies Ditkin's condition, then it induces a homeomorphism between the structure spaces of $A$ and $B$.

Finally, we show that linear isometries between regular uniform algebras are separating. As corollaries, a classical theorem of Nagasawa ([19]) and the Banach-Stone theorem (both for regular uniform algebras) are easily inferred.
\end{abstract}

1. Introduction. The basic problem in the theory of automatic continuity of linear operators consists of giving algebraic conditions on two Banach algebras $\mathscr{A}$ and $\mathscr{B}$ which ensure that every algebra homomorphism $\mathscr{T}: \mathscr{A} \rightarrow \mathscr{B}$ is necessarily continuous. The most important positive result in this context is due to B. E. Johnson ([15]). Every algebra homomorphism of a Banach algebra onto a semisimple Banach algebra is continuous.

In this paper we shall study the automatic continuity of a special type of linear mapping which extends the concept of algebra homomorphism: Let $A$ and $B$ be two Banach algebras. Then a linear map $T$ defined from $A$ into $B$ is said to be separating or disjointness preserving (also called $d$-homomorphisms, Lamperti operators, ...) if $f . g \equiv 0$ implies $T f . T g \equiv 0$, for all $f, g \in A$. Linear maps $T: L_{p}(\mu) \rightarrow L_{p}(\mu)$ with the property that $f . g \equiv 0$ ( $\mu$ a.e.) implies $T f . T g \equiv 0$ ( $\mu$ a.e.) were already considered by Banach [7, p. 175]. In [18], J. Lamperti continued Banach's research and later W. Arendt [5] called such maps Lamperti operators. In the context of vector lattices, disjointness preserving operators $T: E \rightarrow F$ (with $E, F$ vector lattices) are defined to satisfy the implication $|f| \wedge|g|=0 \Rightarrow|T f| \wedge|T g|=0$ and have been widely studied: their multiplicative representation in, for example [1], their spectral properties in [6]; recently, Huijsmans-de Pagter [14] and, independently, Koldunov [17] have shown that the inverse of a disjointness preserving bijection is disjointness preserving under certain general conditions.

Disjointness preserving maps were also considered in [8] for spaces of real or complex-valued continuous functions defined on a compact Hausdorff space with the name of separating maps. The main goal in this context is to prove automatic continuity results for separating maps between different kinds of function spaces of the following type.

TheOREM. ([16] and [10].) A separating bijection $T: C(X) \rightarrow C(Y)$ with $X, Y$ compact (resp. $T: C_{0}(X) \rightarrow C_{0}(Y)$ with $X, Y$ locally compact), is continuous and induces $a$ homeomorphism of $Y$ onto $X$.

$†$ Research partially supported by Generalitat Valenciana, (2223/94).

Glasgow Math. J. 39 (1997) 333-343. 
THEOREM. ([11].) A separating bijection $T: L^{1}\left(G_{1}\right) \rightarrow L^{1}\left(G_{2}\right)$ (with $G_{1}$ and $G_{2}$ locally compact Abelian groups) is continuous and induces a homeomorphism between their dual groups.

Our main result here (§4) will show that a separating bijection $T: A \rightarrow B$ (where $A$ and $B$ are regular semisimple commutative Banach algebras) is automatically continuous whenever $A$ satisfies Ditkin's condition, its inverse is separating and induces a homeomorphism between their structure spaces if also $B$ satisfies such condition. As a consequence (see $[13, \S 39]$ ), the results (op. cit.) in [16], [10] and [11] are extended to a wider class of regular Banach function algebras that includes, for instance, Segal algebras [21] or the Banach sequence algebras $l^{p}(\mathbf{N}), p \in(0, \infty)$, in [9]. It is, however, important to remark that a separating map need not be continuous; indeed, $K$. Jarosz proved [16] that, given two compact spaces $X$ (infinite) and $Y$, there always exists a discontinuous separating map defined from $C(X)$ into $C(Y)$; see also [8].

In $\S 5$, we show, in the spirit of $[12$, Corollary 4.3$]$ and [10, Theorem 4], that linear isometries between regular uniform algebras are separating. As a corollary of this, we easily derive a classical result of Nagasawa [19] and the Banach-Stone theorem for regular uniform algebras.

The author is indebted to Professor Salvador Hernández for helpful conversations from which this work stems and to the referee for suggesting improvements in the presentation.

2. Preliminaries. Let $A$ be a commutative Banach algebra which may or may not have an identity element. Let $X$ denote the set of all complex-valued multiplicative linear functionals on $A$. We can identify $X$ with a subset of the unit sphere of the conjugate space $A^{\prime}$ of $A$. Endowed with the Gelfand topology, that is, the weak-star topology that it inherits from $A^{\prime}, X$ is said to be the structure space or the maximal ideal space of $A$. If $A$ has a unit, then its structure space is a compact Hausdorff space. Otherwise, it is a locally compact Hausdorff space.

The Gelfand transform of $f \in A$ is the complex-valued function $\hat{f}$ on $X$ defined by $\hat{f}(x)=x(f)$. The Gelfand transform is a homomorphism of $A$ onto a point-separating subalgebra $A$ of $C_{0}(X)$ (the Banach algebra of all complex-valued continuous functions on $X$ which are zero at infinity provided with the supremum norm $\|\cdot\|_{\infty}$ ). In addition, $\|\hat{f}\|_{\infty} \leq\|f\|_{A}$ for every $f \in A$, which yields the continuity of the Gelfand transform.

It is said that $A$ is semisimple if the set $\cap\{\operatorname{ker}(x): x \in X\}$ is $\{0\}$. In this case, the Gelfand transform is injective. If $A$ is a semisimple commutative Banach algebra, then $\left(A,\|\cdot\|_{A}\right)$ and the Banach function algebra $\left(\hat{A},\|\cdot\|_{A}\right)$ are indistinguishable as Banach algebras. Indeed, the class of semisimple commutative Banach algebras coincides with the class of Banach function algebras.

Let us now consider the hull-kernel topology on $X$. In general, this topology differs from the Gelfand topology. They coincide if the hull-kernel topology is Hausdorff, which occurs if and only if for every closed subset $E$ of $X$ and every $x \in X \backslash E$, there is $f \in A$ such that $\hat{f}(x) \neq 0$, while $\hat{f}$ vanishes on $E$. If a Banach algebra $A$ has one of these equivalent properties, then $A$ is said to be regular.

Associated with a separating map $T: A \rightarrow B$, we can define a linear mapping $\hat{T}: \hat{A} \rightarrow \hat{B}$ as $\hat{T}(\hat{f}):=\widehat{T(f)}$, for every $f \in A$. If $A$ and $B$ are semisimple, then it is easy 
to check that $T$ is a separating map if and only if $\hat{T}$ is separating. In like manner, $T$ is injective (resp. onto) if and only if $\hat{T}$ is injective (resp. onto).

$\mathbf{N}$ (resp. $\mathbf{R}, \mathbf{C}$ ) will stand for the set of all natural numbers (resp. real, complex numbers). If $X$ is locally compact, then $X^{*}$ will denote its Alexandroff compactification; that is, $X^{*}=X \cup\{\infty\}, \infty$ being an ideal point with a neighbourhood consisting of all sets in $X^{*}$ whose complement is compact in $X$. If $f \in C(X)$ (the linear space of all complex-valued continuous functions on $X$ ), the cozero of $f$ is the set $\operatorname{coz}(f):=$ $\{x \in X: f(x) \neq 0\}$ and $\operatorname{supp}(f)$ will denote the support of $f$; i.e., the closure of $\operatorname{coz}(f)$. When $U$ is any subset of $X$, we shall denote by $\operatorname{int}(U)$ the interior of $U$ and by $\operatorname{cl}(U)$ the closure of $U$ in $X . f_{\mid U}$ stands for the restriction of $f$ to $U$, for any $f \in C(X)$.

Finally, if $y \in Y$, let $T^{t} y^{t}: \hat{A} \rightarrow \mathbf{C}$ be defined as $T^{t} y^{t}(\hat{f})=\hat{T}(\hat{f})(y)$, for all $f \in \hat{A}$.

3. Previous results. In the sequel we shall use the following two results concerning regular commutative Banach algebras (see e.g.; [13], §39]).

Propostrion 1. Let $\mathscr{A}$ be a regular commutative Banach algebra. Let $E$ be a compact subset of its structure space and disjoint from a closed subset $F$. Then there is $f \in \mathscr{A}$ such that $\hat{f}=1$ on $E$ and $\hat{f}=0$ on $F$.

Proposition 2. If $\mathscr{A}$ is a regular commutative Banach algebra with identity and $\left\{U_{j}\right\}_{j=1}^{n}$ is an open cover of its structure space, then there are $f_{j} \in \mathscr{A}$ such that the support of $\hat{f}_{j}$ is contained in $U_{j}$, and $\sum_{j=1}^{n} \hat{f}_{j}=1$.

Definition 1. Let $A$ and $B$ be semisimple commutative Banach algebras, $X$ and $Y$ their respective structure spaces and $T: A \rightarrow B$ a separating map. An open subset $V$ of $X^{*}$ is said to be a vanishing set for $T^{\prime} y^{\prime}, y \in Y$, if for all $\hat{f} \in \hat{A}$ such that $\operatorname{coz}(\hat{f}) \subseteq V$, then $T^{\prime} y^{\prime}(\hat{f})=0$.

$Y_{0}$ will stand for the elements $y \in Y$ such that there exists $f_{y} \in A$ with $\hat{T}\left(\hat{f}_{y}\right)(y) \neq 0$.

Fix $y \in Y_{0}$. Then we define the set

$$
\text { supp } T^{t} y^{t}:=X^{*} \backslash \bigcup\left\{V \subset X^{*}: V \text { is a vanishing set for } T^{t} y^{t}\right\} \text {. }
$$

Lemma 1. With the same notation as in Definition 1 , let $y \in Y_{0}$. Then the set supp $T^{t} y^{t}$ contains, at most, one element. If, in addition, $A$ is regular, then supp $T^{t} y^{t}$ is a singleton.

Proof. Let us suppose that there exist two distinct elements $x_{1}$ and $x_{2}$ in supp $T^{t} y^{t}$. Let $U$ and $V$ be two disjoint open neighbourhoods of $x_{1}$ and $x_{2}$, respectively. Then there exist $f, g \in A$ such that

$$
\hat{T}(\hat{f})(y) . \hat{T}(\hat{g})(y) \neq 0
$$

with $\operatorname{coz}(\hat{f}) \subset U$ and $\operatorname{coz}(\hat{g}) \subset V$, which contradicts the separating property of $\hat{T}$.

Let us now assume that $A$ is regular. It is well known that we can embed $A$ as an ideal in a regular commutative Banach algebra $A_{1}$ with identity. Furthermore, $X$ is a locally compact subspace of the (compact) structure space $X_{1}$ of $A_{1}$. Indeed, $X_{1}=X^{*}$.

Let us suppose that supp $T^{t} y^{t}$ is empty. In this case, we have that

$$
X^{*}=\bigcup\left\{V \subset X^{*}: V \text { is a vanishing set for } T^{t} y^{t}\right\} .
$$


Since $X^{*}$ is a compact set, there exist finitely many vanishing sets $U_{1}, U_{2}, \ldots, U_{n}$ of $T^{t} y^{t}$ such that $X^{*}=U_{1} \cup \ldots \cup U_{n}$. By Proposition 2, there are $f_{j} \in A_{1}$ such that the support of $\hat{f}_{j}$ is contained in $U_{j}$, and $\sum_{j=1}^{n} \hat{f}_{j}=1$. Hence, since $A$ is an ideal in $A_{1}$, we have that $\hat{f}=\sum_{j=1}^{n} \hat{f}_{j} . \hat{f}$, for all $f \in A$. Furthermore, since $\operatorname{coz}\left(\hat{f}_{j}, \hat{f}\right) \subset U_{j}$, we deduce from the definition of a vanishing set that $\hat{T}\left(\hat{f}, \hat{f}_{j}\right)(y)=0$, for $j=1, \ldots, n$. Thus $\hat{T}(\hat{f})(y)=0$, for all $f \in A$, which contradicts the fact that $y \in Y_{0}$.

REMARK 1. (1) A simple example shows that, without the assumption of regularity, supp $T^{t} y^{t}$ may be empty. Let $A(\mathscr{D})$ be the disc algebra, $\mathscr{D}=\{y \in \mathbf{C}:|y| \leq 1\}$. It is well known that $A(\mathscr{D})$ is a non-regular Banach function algebra on $\mathscr{D}$. Let us define $T(f)=f$, for all $f \in A(\mathscr{D})$. If we fix any $y \in \operatorname{int}(\mathscr{D})$, then it is clear that the only possibility would be supp $T^{t} y^{t}=y$, but, by the analytic identity theorem, we can find an open neighbourhood $V$ of $y$ such that $\operatorname{coz}(f) \cap(\mathscr{D} \backslash V) \neq \varnothing$, for all $f \in A(\mathscr{D})$. This and the paragraph above show that $\operatorname{supp} T^{t} y^{t}=\varnothing$ for all $y \in \operatorname{int}(\mathscr{D})$.

(2) It is straightforward to check that $\operatorname{supp} T^{t} y^{t}=\left\{x \in X^{*}\right.$ : for any $X^{*}-$ neighbourhood $U$ of $x$, there exists $f \in A$ such that $\hat{T} \hat{f}(y) \neq 0$ and $\operatorname{coz}(\hat{f}) \subset U \cap X\}$.

Definition 2. Let $A$ and $B$ be regular semisimple commutative Banach algebras, $X$ and $Y$ their respective structure spaces and $T: A \rightarrow B$ a separating map. The above lemma lets us define a mapping $h: Y_{0} \rightarrow X^{*}$ with $h(y):=\operatorname{supp} T^{t} y^{t}, y \in Y_{0}$. We call $h$ the support map of $\hat{T}$.

Proposition 3. With the same notation as in Definition 2, let $U$ be an open subset of $X^{*}$ and suppose that $f \in A$. Then the following statements hold.

(1) The support map $h$ of $\mathcal{T}$ is continuous.

(2) $\hat{f}_{\mid \cup \cap X} \equiv 0$ implies that $\uparrow \hat{f}_{\left.\right|^{-1}(U)} \equiv 0$.

(3) $h(\operatorname{coz}(\hat{T} \hat{f})) \subset \operatorname{cl}_{X^{*}}(\operatorname{coz}(\hat{f}))$.

(4) If $T$ is injective, then $h\left(Y_{0}\right)$ is a dense subset of $X^{*}$.

Proof. It can be found basically in [1, Proposition 3.1].( See also [8] or [16].)

Definition 3. $([13,39.23])$ A commutative Banach algebra $\mathscr{A}$ is said to satisfy Ditkin's condition if, for every $f \in \mathscr{A}$ and $x \in X$ such that $\hat{f}(x)=0$, there exists a sequence $\left\{f_{n}\right\} \subset \mathscr{A}$ and open neighbourhoods $V_{n}$ of $x$ such that $\hat{f}_{n \mid v_{n}} \equiv 0$, for all $n \in \mathbf{N}$, and $\lim \left\|f . f_{n}-f\right\|_{\mathscr{A}}=0$.

$n$ Furthermore, if $\mathscr{A}$ does not have a unit, then, for every $f \in \mathscr{A}$, there must exist a sequence $\left\{f_{n}\right\} \subset \mathscr{A}$ such that $\left\{\hat{f}_{n}\right\} \subset C_{00}(X)$ (the subspace of $C_{0}(X)$ consisting of functions with compact support) and $\lim _{n}\left\|f . f_{n}-f\right\|_{\infty}=0$.

Proposition 4. Let $A$ and $B$ be regular semisimple commutative Banach algebras, $X$ and $Y$ their respective structure spaces and $T: A \rightarrow B$ a separating map. Let $y \in Y_{0}$.

(1) If $A$ satisfies Ditkin's conditon and $T^{t} y^{t}:\left(\hat{A},\|\cdot\|_{A}\right) \rightarrow \mathbf{C}$ is a continuous map, then $h(y) \in X$.

(2) If $T^{t} y^{t}:\left(\hat{A},\|\cdot\|_{\infty}\right) \rightarrow \mathrm{C}$ is a continuous map, then $h(y) \in X$. 
Proof. (1) Let us suppose that $h(y) \notin X$; that is, $h(y)=\infty$. Let $f \in A$. Since $A$ satisfies Ditkin's condition, there is a sequence $\left\{f_{n}\right\} \subset A$ such that $\left\{\hat{f}_{n}\right\} \subset C_{00}(X)$ and $\lim _{n}\left\|f \cdot f_{n}-f\right\|_{\mathscr{A}}=0$, which implies that $\left\{\hat{f} \cdot \hat{f}_{n}\right\}$ converges to $\hat{f}$ in $\left(\hat{A},\|\cdot\|_{A}\right)$.

Furthermore, $\left(\hat{f}_{n} . \hat{f}\right) \equiv 0$ on $\left(X^{*} \backslash \operatorname{cl}\left(\operatorname{coz}\left(\hat{f}_{n}\right)\right)\right) \cap X$ and $y \in h^{-1}\left(X^{*} \backslash \operatorname{cl}\left(\operatorname{coz}\left(\hat{f}_{n}\right)\right)\right)$, since $h(y)=\infty$ and $f_{n} \in C_{00}(X)$, for every $n \in \mathbf{N}$. Consequently, from Proposition 3 , we have that $\hat{T}\left(\hat{f}_{n}, \hat{f}\right)(y)=0$, for every $n \in \mathbf{N}$.

As $T^{t} y^{t}:\left(\hat{A},\|\cdot\|_{A}\right) \rightarrow \mathbf{C}$ is a continuous mapping, the sequence $\left\{T^{t} y^{t}\left(\hat{f}_{n} . \hat{f}\right)\right\}$ converges to $T^{\prime} y^{t}(\hat{f})$. Consequently, since $T^{t} y^{t}\left(\hat{f}_{n}, \hat{f}\right)=0$, for all $n \in \mathbf{N}$, we infer that $T^{\prime} y^{\prime}(\hat{f})=0$.

Summing up, $T^{t} y^{t}(f)=0$, for every $f \in A$, which contradicts the fact that $y \in Y_{0}$. (See Definition 1.)

(2) Let us suppose that $h(y) \notin X$; that is, $h(y)=\infty$. Since $A \subseteq C_{0}(X)$, we can consider the following neighbourhood of $h(y)$ for any $f \in A$ :

Also let

$$
U_{n}:=\{x \in X:|\hat{f}(x)| \leq 1 / n\}
$$

$$
K_{n}:=\{x \in X:|\hat{f}(x)| \geq 2 / n\},
$$

for $n \in \mathbf{N}$. From the definition of $C_{0}(X)$, each $K_{n}$ is a compact subset of $X$.

By Proposition 1, there exists $g_{n} \in A$ such that $\hat{g}_{n \mid K_{n}} \equiv 1$ and $\hat{g}_{n \mid U_{n}} \equiv 0$, for all $n \in \mathbf{N}$. Hence, the sequence $\left\{\hat{g}_{n}, \hat{f}\right\}$ converges to $\hat{f}$ in $\left(\hat{A},\|\cdot\|_{\infty}\right)$ since $\left\|\hat{f}-\left(\hat{g}_{n} \cdot \hat{f}\right)\right\|_{\infty}<\frac{2}{n}$, for all $n \in \mathbf{N}$.

Furthermore, from Proposition 3, we have that $\hat{T}\left(\hat{g}_{n} \cdot \hat{f}\right)(y)=0$, for all $n \in \mathbf{N}$, since $\left(\hat{g}_{n}, \hat{f}\right)_{U_{n} \cap X} \equiv 0$ and $y$ belongs to $h^{-1}\left(U_{n}\right)$. The rest of the proof follows from the same arguments as in (1) above.

Remark 2. Let $A$ and $B$ be regular semisimple commutative Banach algebras, $X$ and $Y$ their respective structure spaces and $T: A \rightarrow B$ a separating map. Let us define a continuous map $\omega: h^{-1}(X) \subseteq Y_{0} \rightarrow \mathrm{C}$ as follows. Given $y \in h^{-1}(X)$, let $U$ be a relatively compact open neighbourhood of $h(y)$ and let $e_{(y, U)}$ be any function in $A$ such that $\hat{e}_{(y, U)}=1$ on $U$. These functions are provided by Proposition 1 . Let us now define

$$
\omega(y):=\hat{T}\left(\hat{e}_{(y, U)}\right)(y)
$$

By Proposition 3, it is easy to check that the definition of $\omega$ does not depend on the choice of the function $e_{(y, U)}$; i.e., if $\hat{e}_{(y, U)}^{\prime} \equiv 1$ on $U$, then $\hat{T}\left(\hat{e}_{(y, U)}^{\prime}\right)(y)=\hat{T}\left(\hat{e}_{(y, U)}\right)(y)$. On the other hand, if $V$ is another relatively compact neighbourhood of $h(y)$ and $e_{(y, V)}$ is as above, then $\hat{T}\left(\hat{e}_{(y, U)}\right)=\hat{T}\left(\hat{e}_{(y, V)}\right)$ on $h^{-1}(U \cap V)$, by Proposition 3 .

Hence, the mapping $\omega$ is well defined and, given that for all $y \in h^{-1}(X)$ this mapping is equal to $\hat{T}\left(\hat{e}_{(y, U)}\right)$ on $h^{-1}(U)$ (which is a neighbourhood of $y$ ), we infer that $\omega$ is a continuous mapping.

Definition 4. Let $A$ and $B$ be regular semisimple commutative Banach algebras, $X$ and $Y$ their respective structure spaces and $T: A \rightarrow B$ a separating map. We denote by $Y_{c}$ the subset of $Y_{0}$ consisting of all $y$ such that $T^{t} y^{t}:\left(A,\|.\|_{\infty}\right) \rightarrow \mathbf{C}$ is continuous and by $Y_{d}$ the complement of $Y_{c}$ in $Y_{0}$. 
Proposrtion 5. With the same notation as in Definition 4, let $y \in h^{-1}(X)$. The following statements are equivalent.

(1) The map $T^{t} y^{t}:\left(\hat{A},\|\cdot\|_{\infty}\right) \rightarrow \mathrm{C}$ is continuous; i.e., $y \in Y_{c}$.

(2) $\hat{T}(\hat{f})(y)=\omega(y) \cdot \hat{f}(h(y))$, for every $f \in A$.

Proof. (1) $\Rightarrow(2)$. First we claim that if $\hat{f}(h(y))=0, f \in A$, then $\hat{T}(\hat{f})(y)=0$. To this end, and since $\hat{f}(h(y))=0$ and $\hat{f}$ is continuous, there exist, for every $n \in \mathbf{N}$, open neighbourhoods $U_{n}$ of $h(y)$ such that $\sup \left\{|\hat{f}(x)|: x \in \operatorname{cl}\left(U_{n}\right)\right\}<\frac{1}{n}$. Let us consider a relatively compact open neighbourhood $V_{n}$ of $h(y)$ such that $\operatorname{cl}\left(V_{n}\right) \subset U_{n}$, for all $n \in \mathbf{N}$. By Proposition 1, we can choose $g_{n} \in A$ such that $\hat{g}_{n|c| c\left(V_{n}\right)} \equiv 1$ and $\hat{g}_{n} \equiv 0$ outside $U_{n}$. Clearly the sequence $\left\{\hat{f} . \hat{g}_{n}\right\}$ converges to the function 0 and $\hat{f} . \hat{g}_{n} \equiv \hat{f}$ on $V_{n}$, for all $n \in \mathbf{N}$. Hence, by Proposition 3, we have that $\hat{T}(\hat{f})(y)=\hat{T}\left(\hat{f} \cdot \hat{g}_{n}\right)(y)$ and consequently, since $T^{t} y^{t}$ is a continuous mapping, $\hat{T}(\hat{f})(y)=0$.

On the other hand, let us suppose that $\hat{f}(h(y)) \neq 0$. Define the function $g:=$ $f-(\hat{f}(h(y))) \cdot e_{(y, V)}\left(e_{(y, V)}\right.$ being as in the above remark). Since $\hat{T}$ is linear and $\hat{g}(h(y))=0$, we infer that $\hat{f}(\hat{f})(y)=\omega(y) \cdot \hat{f}(h(y))$, for all $f \in A$.

(2) $\Rightarrow(1)$. Clear hold.

Proposition 6. With the same notation as in Definition 4, the following statements

(1) $Y_{c}$ is closed in $h^{-1}(X)$.

(2) $h\left(Y_{d}\right)$ is a subset of limit points of $X^{*}$.

(3) If $A$ satisfies Ditkin's condition, then the $\operatorname{set} h\left(Y_{d}\right) \cap \operatorname{int}(K)$ is finite, for every compact subset $K$ of $X$.

Proof. (1) It is apparent, by Proposition 4, that $Y_{c} \subseteq h^{-1}(X)$. Let us consider a net $\left\{y_{\alpha}\right\}$ in $Y_{c}$ that converges to $y \in h^{-1}(X)$. By Proposition 5, $\hat{T}(\hat{f})\left(y_{\alpha}\right)=\omega\left(y_{\alpha}\right) \cdot \hat{f}\left(h\left(y_{\alpha}\right)\right)$, for every $\alpha$ and every $f \in A$. Since $\omega, \hat{f} \circ h$, and $\hat{T}(\hat{f})$ are continuous mappings, it is clear that $\hat{T}(\hat{f})(y)=\omega(y) . \hat{f}(h(y))$, for every $f \in A$; that is, $y \in Y_{c}$ by Proposition 5 .

(2) If $h(y)=\infty$, then $h(y)$ is a limit point of $X^{*}$. Let us now see that if $h(y) \in X$ is isolated in $X, y \in Y$, then $T^{t} y^{t}:\left(\hat{A},\|\cdot\|_{\alpha}\right) \rightarrow \mathbf{C}$ is a continuous map; that is, $y \in Y_{c}$. Given $f \in A$, let us define the map $\hat{g}:=\hat{f}(h(y)) \cdot \hat{e}_{(y, U)}$, for any relatively compact open neighbourhood $U$ of $y$, as in Remark 2. As $\hat{f}_{\mid\{h(y)\}} \equiv \hat{g}_{\mid\{h(y)\}}$, we have that $\hat{T}(\hat{f})(y)=\hat{T}$ $(\hat{g})(y)$, by Proposition 3. Hence, $T^{t} y^{t}(\hat{f})=\omega(y) . \hat{f}(h(y))$, for every $f \in A$, which implies that $T^{i} y^{t}:\left(\hat{A},\|.\|_{\infty}\right) \rightarrow \mathbf{C}$ is continuous, by Proposition 5.

(3) Let us suppose, contrary to what we claim, that there exists a sequence $\left\{h\left(y_{n}\right)\right\}$ of distinct elements of $\operatorname{int}(K)$ such that $y_{n} \in Y_{d}$, for every $n \in \mathbf{N}$. As $K$ is a normal space, we can assume, by taking a subsequence if necessary, that $\left\{U_{n}\right\}$ is a sequence of pairwise disjoint open subsets of $K$ such that $h\left(y_{n}\right) \in U_{n} \subset K$, for every $n \in \mathbf{N}$. Let $V_{n}$ be a closed neighbourhood of $h\left(y_{n}\right)$ with $V_{n} \subset U_{n}$, for every $n \in \mathbf{N}$.

Fix $m \in \mathbf{N}$. By Proposition 5, since the map $T^{t} y_{m}^{t}:\left(\hat{A},\|\cdot\|_{\infty}\right) \rightarrow \mathbf{C}$ is discontinuous, there exists a function $f_{m} \in A$ with $\hat{T}\left(\hat{f}_{m}\right)\left(y_{m}\right) \neq \omega\left(y_{m}\right) \cdot \hat{f}_{m}\left(h\left(y_{m}\right)\right)$. Let us now define the function

$$
\hat{g}_{m}:=\hat{f}_{m}-\left(\hat{f}_{m}\left(h\left(y_{m}\right)\right)\right) \cdot \hat{e}_{\left(y_{m}, V\right)}
$$


where $\hat{e}_{\left(y_{m}, V\right)}$ is as in Remark 2 and $K \subseteq V$. Hence, $\hat{T}\left(\hat{g}_{m}\right)\left(y_{m}\right) \neq 0$ and $\hat{g}_{m}\left(h\left(y_{m}\right)\right)=0$. Since $\hat{T}$ is a linear map, we shall assume without loss of generality that $\left|\hat{T}\left(\hat{g}_{m}\right)\left(y_{m}\right)\right|>m$. Since $A$ satisfies Ditkin's condition, there exists $k_{m} \in A$ such that $\hat{k}_{m \mid v_{m}} \equiv 0$ and $\left\|\varphi_{m}\right\|_{A}<$ $\frac{1}{m^{2}}$, where $\varphi_{m}:=g_{m}-g_{m} \cdot k_{m}$. Furthermore, as $\hat{\varphi}_{m} \equiv \hat{g}_{m}$ on $V_{m}$, we have, by Proposition 3,

$$
\left|\hat{T}\left(\hat{\varphi}_{m}\right)\left(y_{m}\right)\right|=\left|\hat{T}\left(\hat{g}_{m}\right)\left(y_{m}\right)\right|>m \text {. }
$$

Let us now define $\varphi:=\sum_{n \in \mathbb{N}} \varphi_{n}$. Since $\left\|\varphi_{n}\right\|_{A}<\frac{1}{n^{2}}$, for all $n \in \mathbf{N}$, and $A$ is a Banach space, we infer that $\varphi$ belongs to $A$. From the fact that the Gelfand transform is a linear continuous mapping, we deduce that $\hat{\varphi}=\sum_{n \in N} \hat{\varphi}_{n}$.

On the other hand, since the family $\left(U_{n}\right)_{n \in N}$ is pairwise disjoint and $\operatorname{coz}\left(\hat{\varphi}_{n}\right) \subset U_{n}$, for all $n \in \mathbf{N}$, the separating property of $\hat{T}$ yields $T\left(\hat{\varphi}_{n}\right)_{n^{-1}\left(U_{n^{\prime}}\right)} \equiv 0$, for $n \neq n^{\prime}\left(n, n^{\prime} \in \mathbf{N}\right)$. Thus, $\left|\hat{T}\left(\hat{\varphi}_{n}\right)\left(y_{n}\right)\right|=\left|\hat{T}(\hat{\varphi})\left(y_{n}\right)\right|>n$, for every $n \in \mathbf{N}$, which is a contradiction since $\hat{T}(\hat{\varphi})$ is bounded.

\section{Main results.}

THEOREM 1. Let $A$ and $B$ be regular semisimple commutative Banach algebras, $X$ and $Y$ their respective structure spaces and $T: A \rightarrow B$ a separating bijection. Assume further that A satisfies Ditkin's condition.

(1) $T$ is continuous.

(2) $T^{-1}$ is separating.

(3) If also $B$ satisfies Ditkin's condition, then $X$ and $Y$ are homeomorphic.

Proof. (1) Let us first note that $Y=Y_{0}$ since $T$ is onto. Since $Y_{c} \cup Y_{d}=Y$, then $h\left(Y_{c}\right) \cup h\left(Y_{d}\right)=h(Y)$. As $T$ is injective, $h(Y)$ is dense in $X^{*}$, by Proposition 3. Hence, given $x \in X$ and a compact neighbourhood $U$ of $x$, we have, by Proposition 6, $h\left(Y_{c}\right) \cap U \neq \varnothing$. That is, $h\left(Y_{c}\right)$ is dense in $X^{*}$.

Now, if $y \in Y_{c}$, then $\hat{T}(\hat{f})(y)=\omega(y) \cdot \hat{f}(h(y))$, for all $f \in A$, which implies, since $\hat{T}$ is onto and $\hat{A}$ separates the points of $X$, that $\omega(y) \neq 0$. Hence, if $\hat{T}(\hat{f})_{\left.\right|_{\mathrm{Y}}} \equiv 0$, for some $f \in A$, then $\hat{f}_{\mid h\left(Y_{c}\right)} \equiv 0$. Furthermore, we know, by the above paragraph and Proposition 4, that $h\left(Y_{c}\right)$ is dense in $X$. As a consequence, if $\hat{T}(\hat{f})_{\mid Y_{c}} \equiv 0$, then $\hat{f} \equiv 0$.

Next, we claim both that $Y_{c}=h^{-1}(X)$ and that $Y_{c}$ is dense in $Y$. To this end, suppose that there exists $y \in h^{-1}(X) \backslash Y_{c}$. As $Y_{c}$ is, from Proposition 6, a closed subset of $h^{-1}(X)$, there exists, by Proposition 1 and the surjectivity of $\hat{T}$, a function $g \in A$ such that $\hat{T}(\hat{g})_{\mid Y_{c}} \equiv 0$ and $\hat{T}(\hat{g})(y)=1$. This contradicts the above paragraph. In like manner we prove that $Y_{c}$ is dense in $Y$.

Finally, in order to prove the continuity of $T$, we shall prove that it has a closed graph. Let us suppose that the sequences $\left\{f_{n}\right\} \subset A$ and $\left\{T\left(f_{n}\right)\right\}$ converge to $f \in\left(A,\|\cdot\|_{A}\right)$ and $g \in\left(B,\|\cdot\|_{B}\right)$, respectively. Then, given any $y \in Y$, the sequence $\left\{\hat{T}\left(\hat{f}_{n}\right)(y)\right\}$ converges to $\hat{g}(y)$. On the other hand, given any $x \in X$, the sequence $\left\{\hat{f}_{n}(x)\right\}$, converges to $\{\hat{f}(x)\}$. Consequently, for any $y \in Y_{c}$, the sequence $\left\{\hat{T}\left(\hat{f}_{n}\right)(y)\right\}$, that is to say $\left\{\omega(y) . \hat{f}_{n}(h(y))\right\}$, converges to $\hat{T}(\hat{f})(y)=\omega(y) \cdot \hat{f}(h(y))$. Thus, $\hat{T}(\hat{f})=\hat{g}$ on $Y_{c}$, which is dense in $Y$. This implies that $\hat{T}(\hat{f})=\hat{g}$ and, consequently, $T(f)=g$. Finally, since $A$ and $B$ are Banach spaces, the Closed Graph Theorem yields the continuity of $T$. 
(2) Let us first prove that the inverse of $\hat{T}, \hat{T}^{-1}: \hat{B} \rightarrow \hat{A}$, is also a separating bijection. Let $\hat{g}_{1}$ and $\hat{g}_{2}$ be two elements of $\hat{B}$ such that $\hat{g}_{1} \cdot \hat{g}_{2}=0$. Let $\hat{f}_{1}, \hat{f}_{2} \in \hat{A}$ such that $\hat{T} \hat{f}_{1}=\hat{g}_{1}$ and $\hat{T} \hat{f}_{2}=\hat{g}_{2}$. Since $\omega$ is non-vanishing on $Y_{c}$ (see (1) above), we deduce that $\hat{f}_{1} \cdot \hat{f}_{2}=0$ on $h\left(Y_{c}\right)$, which is dense in $X$. As a consequence, $\hat{f}_{1} \cdot \hat{f}_{2}=0$ on the whole of $X$. Hence, both $\hat{T}^{-1}$ and, consequently, $T^{-1}: B \rightarrow A$ are separating maps.

(3) Since $T$ is continuous, it is a routine matter to verify that the map $T^{t} y^{t}:\left(\hat{A},\|\cdot\|_{A}\right) \rightarrow \mathrm{C}$ is continuous, for every $y \in Y$. Thus, by Proposition 4 , we know that $h(y) \in X$, for every $y \in Y$. Therefore, since $h^{-1}(X)=Y_{c}$ (see (1) above), we infer that $Y=h^{-1}(X)=Y_{c}$ and, consequently, $T^{t} y^{t}$ is a weighted composition map, for all $y \in Y$. (See Proposition 5.)

Next, by (2), we can consider the support map $k: X \rightarrow Y^{*}$ of $\hat{T}^{-1}$. Of course, $k$ is continuous and its range is dense in $Y^{*}$, by Proposition 3. Furthermore, by (1) and Proposition 4, we know that $\left(T^{-1}\right)^{t} x^{t}$ is a weighted composition map for all $x \in X$ and $k(X) \subset Y$.

Let us now fix $y \in Y$. We claim that $k(h(y))=y$. Assume, contrary to what we claim, that $k(h(y)) \neq y$. Then there exist disjoint compact neighbourhoods $U$ and $V$ of $k(h(y))$ and $y$, respectively. By Remark $1(2)$, there exists a function $g_{0} \in B$ such that $\operatorname{coz}\left(\hat{g}_{0}\right) \subseteq U$ and $\hat{T}^{-1}\left(\hat{g}_{0}\right)(h(y)) \neq 0$. This implies, by Proposition $3(3)$, that $y \notin \operatorname{cl}\left(\operatorname{coz}\left(\hat{g}_{0}\right)\right)$ and $k(h(y)) \in \operatorname{cl}\left(\operatorname{coz}\left(\hat{g}_{0}\right)\right)$. Let $g_{1} \in B$ (see Proposition 1) such that $\hat{g}_{1}(y) \neq 0$ and $\hat{g}_{1_{1 \cos (\hat{\beta})}} \equiv 0$. Consequently, we have that $\operatorname{coz}\left(\hat{g}_{1}\right) \cap \operatorname{coz}\left(\hat{g}_{0}\right)=\varnothing$ and, since $\hat{T}^{-1}$ is separating, $\operatorname{coz}\left(\hat{T}^{-1}\left(\hat{g}_{1}\right)\right) \cap \operatorname{coz}\left(\hat{T}^{-1}\left(\hat{g}_{0}\right)\right)=\varnothing$. On the other hand, $\operatorname{coz}\left(\hat{T}^{-1}\left(\hat{g}_{0}\right)\right)$ is a neighbourhood of $h(y)$, but if we take any $\hat{f} \in \hat{A}$ such that $\operatorname{coz}(\hat{f}) \subseteq \operatorname{coz}\left(\hat{T}^{-1}\left(\hat{g}_{0}\right)\right)$, then $\operatorname{coz}\left(\hat{g}_{1}\right) \cap$ $\operatorname{coz}(\hat{T}(\hat{f}))=\varnothing$, because $\hat{T}$ is separating. Consequently, $\hat{T}(\hat{f})(y)=0$, since $\hat{g}_{1}(y) \neq 0$. This contradicts Remark 1(2). In like manner, we infer that $h(k(x))=x$, for every $x \in X$; that is, $h$ is a homeomorphism of $Y$ onto $X$.

As a straightforward application of our main results, we have the following result. Let $G_{1}$ and $G_{2}$ be locally compact Abelian groups. Let $\widehat{G_{i}}(i=1,2)$ be the dual group of $G_{i}$. Let us recall that a Segal algebra $S\left(G_{i}\right)$ is a dense ideal in the group algebra $L^{1}\left(G_{i}\right)$ with continuous imbedding, on which $G_{i}$ acts continuously by isometric translations. These algebras were introduced in [20]. Some time later, it was proved in [21] that a Segal algebra $S\left(G_{i}\right)$ is a regular semisimple commutative Banach algebra (with respect to a certain norm), which satisfies Ditkin's condition and whose structure space is $\widehat{G_{i}}$.

Corollary 1. A separating bijection $T: S\left(G_{1}\right) \rightarrow S\left(G_{2}\right)$ is automatically continuous and induces a homeomorphism of $\widehat{G_{2}}$ onto $\widehat{G_{1}}$.

5. Isometries between regular uniform algebras. Let $A$ and $B$ be regular semisimple commutative Banach algebras, and $X$ and $Y$ their respective structure spaces. In this section we shall assume, in addition, that $\left\|f^{2}\right\|_{A}=\|f\|_{A}^{2}$, for every $f \in A$ (resp. $\left\|g^{2}\right\|_{B}=\|g\|_{B}^{2}$, for every $g \in B$ ), which implies that the Gelfand transform is an isometry. Consequently, $A$ (resp. $\hat{B}$ ) is a (uniformly) closed subalgebra of $C_{0}(X)$ (resp. $C_{0}(Y)$ ). In this case, $A$ and $B$ are said to be regular uniform algebras on $X$ and $Y$ respectively. If $\mathscr{X}$ is a compact subspace of $\mathbf{C}$ and we consider the algebra of functions on $\mathscr{X}$ uniformly approximable by rational functions with poles off $\mathscr{X}$, then we have an example of a non-trivial regular uniform algebra. 
On the other hand, let us recall that $x_{0} \in X$ is said to be a strong boundary point for $A$ if, for each open neighbourhood $U$ of $x_{0}$, there is $f \in A$ such that $\left|\hat{f}\left(x_{0}\right)\right|=\|\hat{f}\|_{\infty}=1$ and $|\hat{f}(x)|<1$ for all $x \in X \backslash U$. The set of strong boundary points for $A$ is dense in the Shilov boundary for $A$, which here coincides with its structure space $X$ since $A$ is regular. (See [4].)

Lemma 2. Let $A$ be a regular uniform algebra on $X$. If $x_{0} \in X$ is a strong boundary point for $A$ then, for each open neighbourhood $U$ of $x_{0}$ and $\varepsilon>0$, there is $g \in A$ such that $\left|\hat{g}\left(x_{0}\right)\right|=\|\hat{g}\|_{\infty}=1$ and $|\hat{g}(x)|<\varepsilon$, for all $x \in X \backslash U$.

Proof. Since $X^{*} \backslash\{U\}$ is compact the function $\hat{f}$, which is provided by the definition of strong boundary point, attains a maximum value $s<1$ inside $X^{*} \backslash\{U\}$. Hence there exists $n \in \mathbf{N}$ such that $\left|(\hat{f})^{n}(x)\right|<\varepsilon$, for all $x \in X \backslash U$. Thus, $g:=(f)^{n}$ has the required properties.

In [10] and [12], the authors prove that linear isometries between $C_{00}(X)$-spaces and spaces of vector-valued continuous functions, respectively, are separating. The following result shows that the same is true in the context of regular uniform algebras.

THEOREM 2. Let $A$ and $B$ be regular uniform algebras. If $T$ is a linear isometry of $A$ onto $B$, then $T$ and $T^{-1}$ are separating.

Proof. It is obvious that $\hat{T}$ is a linear isometry of $\hat{A}$ onto $\hat{B}$, since the Gelfand transform is an isometry. Let us suppose that there exist $\hat{f}, \hat{g} \in \hat{A}$ and $y_{1} \in Y$ such that $\hat{f} \cdot \hat{g} \equiv 0$ with $\hat{T}(\hat{f})\left(y_{1}\right) . \hat{T}(\hat{g})\left(y_{1}\right) \neq 0$. Since the set of strong boundary points for $B$ is dense in $Y$, there exists a strong boundary point $y_{0}$ such that $\hat{T}(\hat{f})\left(y_{0}\right)=a \neq 0$ and $\hat{T}(\hat{g})\left(y_{0}\right)=b \neq 0$.

Multiplying by constants if necessary, we shall assume that $a, b \in \mathbf{R},|a| \geq|b|$, and $\|\hat{f}\|_{\infty}=\|\hat{g}\|_{\infty}=1$.

As $a, b$ are reals, we know that $|a|<\max (|a+b|,|a-b|)$. Let us suppose that $|a+b|>|a| \geq|b|$ and let $\lambda \in \mathbf{R}$ such that $0<\lambda<\frac{|a+b|-|a|}{2}$.

We define

$$
\begin{aligned}
& V=\{y \in Y:|\hat{T}(\hat{f})(y)|<|a|+\lambda\}, \\
& W=\{y \in Y:|\hat{T}(\hat{g})(y)|<|b|+\lambda\}
\end{aligned}
$$

and $U=V \cap W$. It is clear that $U$ is an open neighbourhood of $y_{0}$ and, since $y_{0}$ is a strong boundary point for $B$, Lemma 2 ensures that there exists $h \in B$ such that $0 \leq|h| \leq 1$ with $h\left(y_{0}\right)=\frac{a+b}{|a+b|}$ and $h(Y \backslash U)<|a+b|$. Then

$$
\left|\hat{T}(\hat{f})\left(y_{0}\right)+\hat{T}(\hat{g})\left(y_{0}\right)+h\left(y_{0}\right)\right|=\left|a+b+\frac{a+b}{|a+b|}\right|=1+|a+b| .
$$

Hence, $\|\hat{T}(\hat{f})+\hat{T}(\hat{g})+h\|_{\infty} \geq 1+|a+b|$.

Since $\hat{T}$ is onto, there exists $k \in B$ such that $\hat{T}(\hat{k})=\hat{h}$. Hence, since $\hat{T}$ is an isometry, we infer that $\|\hat{f}+\hat{g}+\hat{k}\|_{\infty} \geq 1+|a+b|$. As $\hat{f} \cdot \hat{g} \equiv 0$, we have that $\max \left(\|\hat{f}+\hat{k}\|_{\infty}\right.$, $\left.\|\hat{g}+\hat{k}\|_{\infty}\right) \geq 1+|a+b|$. Therefore, if we assume that $\|\hat{f}+\hat{k}\|_{\infty} \geq 1+|a+b|$, then $\|\hat{T}(\hat{f})+\hat{h}\|_{\infty} \geq 1+|a+b|$. 
Given any element $y$ of $Y$ such that $y \in U$, we have that $|\hat{T}(\hat{f})(y)+h(y)| \leqslant$ $|\hat{T}(\hat{f})(y)|+|\hat{h}(y)|<|a|+\lambda+1<1+|a+b|$. On the other hand, if $y \notin U$, then $|\hat{T}(\hat{f})(y)+\hat{h}(y)| \leq \| \hat{T}(\hat{f})(y)|+| \hat{h}(y)|\leq 1+| a+b \mid$. Hence, for every $y \in Y$, we infer that $|\hat{T}(\hat{f})(y)+h(y)|<1+|a+b|$, which contradicts the above paragraph. This proves that $T$ and, by Theorem $1(2), T^{-1}$ are separating.

In [19], Nagasawa proved that two unital uniform algebras are isometric if and only if they are (algebra) isomorphic. As a corollary of Theorem 2, we easily derive this classical theorem of Nagasawa and the Banach-Stone theorem ([3]) in the setting of regular uniform algebras.

COROLlary 2. Let $A$ and $B$ be regular uniform algebras. Let us suppose that there exists a linear isometry $T$ of $A$ onto $B$.

(1) (Banach-Stone) Their structure spaces are homeomorphic.

(2) ([19]) If $A$ and $B$ have units, they are (algebra) isomorphic.

Proof. (1) Combine Theorems 1(3) and 2. (Note that the hypothesis on Theorem $1(3)$ is redundant whenever $T^{-1}$ is continuous.)

(2) Assume now that $1_{A}$ and $1_{B}$ are the units of $A$ and $B$ respectively. Then one easily checks that the function $\omega$, which we have defined in Remark 2 , turns out to be $\hat{T}\left(\widehat{1_{A}}\right)$, which implies that $\omega \in \hat{B}$. By Proposition $5(2)$, we have that

$$
\omega . \hat{T}(\hat{f} \cdot \hat{g})=\hat{T}(\hat{f}) \cdot \hat{T}(g),
$$

for every $f, g \in A$. Hence, if we now choose $k \in A$ such that $\hat{T}(k)=$ $\widehat{1_{B}}$, then $\omega . \hat{T}\left(\hat{k}^{2}\right)=\widehat{1_{B}}$, which is to say that $\omega^{-1}=\hat{T}\left(\hat{k}^{2}\right)$ belongs to $\hat{B}$. Finally, it is clear that $\omega^{-1} . \hat{T}$ is the desired algebra isomorphism of $\hat{A}$ onto $\hat{B}$.

\section{REFERENCES}

1. Y. Abramovich, Multiplicative representation of disjointness preserving operators. Indag. Math. 45 (1983), 265-279.

2. J. Araujo, E. Beckenstein and L. Narici, Biseparating maps and homeomorphic realcompactifications, J. Math. Anal. Appl. 192 (1995), 258-265.

3. J. Araujo and J. J. Font, Linear isometries between subspaces of continuous functions, Trans. Amer. Math. Soc. 349 (1997), 413-428.

4. J. Araujo and J. J. Font, On Shilov boundaries for subspaces of continuous functions. Topology and Appl. 77 (1997), 79-85. 199-215.

5. W. Arendt, Spectral properties of Lamperti operators. Indiana Univ. Math. J. 32 (1983),

6. W. Arendt and D. R. Hart, The spectrum of quasi-invertible disjointness preserving operators, J. Functional Analysis, 68 (1986), 149-167. 1932).

7. S. Banach, Théorie des opérations linéaires (Chelsea Publishing Company, New York,

8. E. Beckenstein, L. Narici and R. Todd, Automatic continuity of linear maps on spaces of continuous functions, Manuscripta Math. 62 (1988), 257-275. appear.

9. H. G. Dales, Banach algebras and automatic continuity (Oxford University Press), to

10. J. J. Font and S. Hernández, Separating maps between locally compact spaces. Arch. Math. (Basel) 63 (1994), 158-165. 
11. J. J. Font and S. Hernández, Automatic continuity and representation of certain linear isomorphisms between group algebras. Indag. Math. 6(4) (1995), 397-409.

12. S. Hernández, E. Beckenstein and L. Narici, Banach-Stone theorems and separating maps, Manuscripta Math. 86 (1995), 409-416.

13. E. Hewitt and K. A. Ross, Abstract harmonic analysis II (Springer-Verlag, 1963).

14. C. Huijsmans and B. de Pagter, Invertible disjointness preserving operators, Proc. Edinburgh Math. Soc. 37 (1993), 125-132.

15. B. E. Johnson, The uniqueness of the (complete) norm topology, Bull. Amer. Math. Soc. 73 (1967), 537-539.

16. K. Jarosz, Automatic continuity of separating linear isomorphisms, Canad. Math. Bull. 33 (2) (1990), 139-144.

17. A. Koldunov, Hammerstein operators preserving disjointness, Proc. Amer. Math. Soc. 123 (1995), 1083-1095.

18. J. Lamperti, On the isometries of certain function spaces, Pacific J. Math. 8 (1958), 459-466.

19. M. Nagasawa, Isomorphisms between commutative Banach algebras with an application to rings of analytic functions, Kodai Math. Sem. Rep. 11 (1959), 182-188.

20. H. Reiter, Classical harmonic analysis and locally compact groups (Oxford University Press, 1968).

21. L. Y. H. Yap, Every Segal algebra satisfies Ditkin's condition, Studia Math. 40 (1971), 235-237.

Departamento De Matemáticas

UNIVERSidAd JAUME 1

Campus Penyeta

E-12071

CASTELLÓN

SPAIN

E-mail address: font@mat.uji.es 